\title{
Strain-rate dependence for Ni/Al hybrid foams
}

\author{
Anne Jung ${ }^{1, a}$, Martin Larcher ${ }^{2, b}$, Ondrej Jirousek ${ }^{3, c}$, Petr Koudelka ${ }^{3, \mathrm{~d}}$, and George Solomos ${ }^{2, \mathrm{e}}$ \\ ${ }^{1}$ Saarland University, Institute of Applied Mechanics, Campus A4.2, 66123 Saarbrcken, Germany \\ ${ }^{2}$ European Commission, Joint Research Centre (JRC), Institute for the Protection and Security of the Citizen (IPSC), \\ European Laboratory for Structural Assessment, via E. Fermi 2749, 21027 Ispra (VA), Italy \\ ${ }^{3}$ Academy of Sciences of the Czech Republic, v.v.i., Department of Biomechanics, Institute of Theoretical and \\ Applied Mechanics, Prosecka 76, 19000 Prague 9, Czech Republic
}

\begin{abstract}
Shock absorption often needs stiff but lightweight materials that exhibit a large kinetic energy absorption capability. Open-cell metal foams are artificial structures, which due to their plateau stress, including a strong hysteresis, can in principle absorb large amounts of energy. However, their plateau stress is too low for many applications. In this study, we use highly novel and promising Ni/Al hybrid foams which consist of standard, open-cell aluminium foams, where nanocrystalline nickel is deposited by electrodeposition as coating on the strut surface. The mechanical behaviour of cellular materials, including their behaviour under higher strain-rates, is governed by their microstructure due to the properties of the strut material, pore/strut geometry and mass distribution over the struts. Micro-inertia effects are strongly related to the microstructure. For a conclusive model, the exact real microstructure is needed. In this study a micro-focus computer tomography ( $\mu \mathrm{CT}$ ) system has been used for the analysis of the microstructure of the foam samples and for the development of a microstructural Finite Element (micro-FE) mesh. The microstructural FE models have been used to model the mechanical behaviour of the Ni/Al hybrid foams under dynamic loading conditions. The simulations are validated by quasi-static compression tests and dynamic split Hopkinson pressure bar tests.
\end{abstract}

\section{Introduction}

Cellular materials like metal foams are a very interesting class of bionic lightweight materials. Open-cell foams mimic the construction elements of human femur bones or the star fish skeletons. Nature uses these cellular structures as advanced, evolution-optimised lightweight materials. Based on their special microstructure, consisting of a network of interconnected pores, metal foams are able to undergo large deformations at a nearly constant stress, forming a stress plateau $[1,2]$. The stress plateau evolves by the successive collapse of single pore layers. Due to the stress plateau, the foams are able to absorb considerable amounts of kinetic energy by plastic dissipation, whereas metal foams can be used as crash absorbers.

Unfortunately, at the moment, foams have to compete with established materials that were characterized better, up to now and the commercially available foams do not provide the required mechanical properties. In the last few years, there were efforts to improve the mechanical properties of metal foams by coating light substrate foams with hard materials [3-8]. Ni/Al hybrid foams are a highly novel and innovative material. They consist of standard, open-cell aluminium foams with a coating of nanocrystalline nickel. The innovative $\mathrm{Ni} / \mathrm{Al}$ hybrid foams skillfully combine three design strategies of materials

\footnotetext{
a e-mail: anne.jung@mx .uni-saarland.de

b e-mail: martin.larcher@jrc.ec.europa.eu

c e-mail: jirousek@itam.cas.cz

d e-mail: koudelkap@itam.cas.cz

e e-mail: george.solomos@jrc.ec .europa.eu
}

design: cellular lightweight construction, nanotechnology and composites. When these strategies are coupled cleverly and the synergies are used, nanocrystalline coated hybrid foams become an innovative and multifunctional nanomaterial for future applications in the fields of lightweight construction and energy absorption.

Cellular materials are called microheterogeneous materials, because their mechanical behaviour can be described on different scales. For metal foams, three different hierarchical scales can be defined. The macro scale deals with the complete sample or component, the meso scale with several pores and the micro scale describes the mechanical behaviour of the struts. Hence, the global mechanical properties of metal foams strongly depend on the microstructure, especially under higher strain-rates. Under high strain rates, the strut geometry and mass distribution over the struts plays a significant role, since micro-inertia effects are strongly related to these microstructural features. Hence, for conclusive models, especially to describe the global mechanical behaviour of the foams under high strain rates, the real microstructure is needed.

In this study, Ni/Al hybrid foams were experimentally and numerically investigated for their strain-rate dependency. Quasi-static and dynamic compression tests were performed. To determine the microstructure, a micro-focus computer tomography system has been used. Based on the $\mu \mathrm{CT}$ measurements, a microstructural Finite Element (micro-FE) mesh was developed. The microstructural FE models have been used to model the mechanical behaviour of the $\mathrm{Ni} / \mathrm{Al}$ hybrid foams under dynamic 
loading conditions and were validated by the macromechanical experiments.

\section{Materials and methods}

\subsection{Synthesis of the Ni/Al hybrid foams}

Aluminium alloy foams $\left(\mathrm{AlSi}_{7} \mathrm{Mg}_{0.3}\right.$, Celltec Materials, Dresden, Germany) with a pore size of 30 ppi (pores per inch) were investigated. To produce Ni/Al hybrid foams, the aluminium foams were coated with different theoretical overall coating thicknesses ranging from 25 to $75 \mu \mathrm{m}$ nano nickel with a crystallite size of about $50 \mathrm{~nm}$, using direct current electrodeposition. A commercial nickel sulfamate electrolyte (Enthone GmbH, Langenfeld, Germany) with a nickel content of $110 \mathrm{~g} / \mathrm{L}$ nickel was used at a $\mathrm{pH}$ of 3.8 and a temperature of $40^{\circ} \mathrm{C}$. For a qualitatively good coating on the aluminium, the pretreatment steps of pickling and electroless plating preventing the dissolution of the aluminium in the acid nickel electrolyte, have been performed [5]. To guarantee a quite homogeneous coating, a cage-like anode filled with nickel pellets from Ampere $\mathrm{GmbH}$, Dietzenbach, Germany, was used as described by Jung et al. [6-8].

For the $\mu \mathrm{CT}$ measurements, cubic samples with an edge length of $15 \mathrm{~mm}$ were coated with $75 \mu \mathrm{m}$ nickel. According to a bar diameter of $20 \mathrm{~mm}$ for the split Hopkinson pressure bar apparatus, cylindrical samples with a diameter of $20 \mathrm{~mm}$ and a height of $5 \mathrm{~mm}$ were used for the dynamic as well as for the quasi-static compression tests. The foams were coated with 25,50 and $75 \mu \mathrm{m} \mathrm{Ni}$, respectively.

\subsection{Micro-focus computer tomography}

High resolution microCT measurements of the samples were performed using a state-of-the-art DSCT/DECT X-ray imaging device developed in the Centre of Excellence Telč (patent pending, European Patent Office No. 14002662.6-1559 and Industrial Property Office of Czech republic No. PV2013-607). The device (depicted in Fig. 1) was designed to achieve maximum flexibility of the measurement in terms of specimen dimensions, magnification and beam characteristics. For this reason, a flexible modular design based on an orthogonal assembly of two tube-detector imaging pairs with independent geometry setting and a shared rotational stage was mounted on a complex 16-axis CNC positioning system. This enables unprecedented variability of measurement for highly detailed tomographical measurements, which is particularly advantageous in the case of heterogeneous materials with complex microstructures (i.e. composites, foams, stones, etc.).

In this work, samples of the metal foam were irradiated using a reflection type X-ray tube XWT-240-SE (X-ray Worx, Germany; voltage range $20-240 \mathrm{kV}$, minimum focal spot size $5 \mathrm{~mm}$, maximum target power $280 \mathrm{~W}$ ) with voltage and target current set to $80 \mathrm{kV}$ and $300 \mu \mathrm{A}$ resulting in a target power of $24 \mathrm{~W}$. Acquisition was performed using large area GOS scintillator flat panel detectors XRD1622 (PerkinElmer, USA) with an active area $410 \times 410 \mathrm{~mm}$, resolution $2048 \times 2048$ px (pixel size

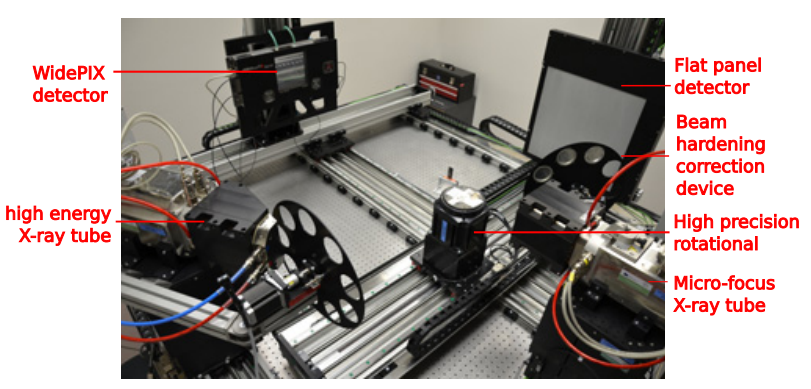

Figure 1. Laboratory setup of DSCT/DECT X-ray imaging device used for the tomography measurements. The sample was fixed on the rotational table in the middle (the sample is not depicted in the figure).

$200 \mathrm{~mm}$ ) at $2 \times 1000 \mathrm{~ms}$ acquisition time. Setup geometry was set to obtain the maximum possible magnification leading to a source-object distance of $79.7 \mathrm{~mm}$ and a source-detector distance of $1345.4 \mathrm{~mm}$, which provided a $16.87 \times$ magnification. Tomographic scanning was performed in 1200 projections with a $0.3^{\circ}$ angular step to improve the accuracy and reliability of the tomographic reconstruction due to the very complex microstructure of the investigated material.

The linearization of the attenuation range (beam hardening correction) [9] was applied to all acquired radiographic projections. During the correction procedure, a set of aluminium calibrators (thicknesses $0.4-2 \mathrm{~mm}$ ) covering the whole attenuation range of the specimens in their widest part was irradiated. The image data were corrected using the attenuation curve fitted through the measured Al calibrators' thicknesses.

\subsection{Quasi-static and dynamic compression tests}

Quasi-static compression tests were performed on $30 \mathrm{ppi}$ aluminium and $\mathrm{Ni} / \mathrm{Al}$ hybrid foams using an INSTRON 4204 universal testing machine at a strain-rate of about $5 \times 10^{-3} \mathrm{~s}^{-1}$

The dynamic compression tests were conducted using a classical split Hopkinson pressure bar (SHPB) apparatus. The cylindrical bars are made of Zicral (AlZn 7075) to guarantee a comparable mechanical impedance between the bars and the sample. Using the SHPB setup, strainrates of up to $5000 \mathrm{~s}^{-1}$ were applied to cylindrical samples (diameter $=20 \mathrm{~mm}$; height $=5 \mathrm{~mm}$ ) of pure aluminium foams and $\mathrm{Ni} / \mathrm{Al}$ hybrid foams with a pore size of $30 \mathrm{ppi}$ and varying coating thicknesses.

\subsection{Dynamic material modelling}

The strain-rate dependency of the foam has also be investigated by using numerical methods with the explicit finite element code EUROPLEXUS (http://www-epx. cea.fr/). This is done using a micro model, which means that the microstructure of the material is dissolved in order to obtain the material behaviour on a macro level. This kind of investigations are very powerful as they can be used as a kind of virtual laboratory testing in order to investigate the material behaviour. They need a strong computational power and adapted material laws for the microstructure. 


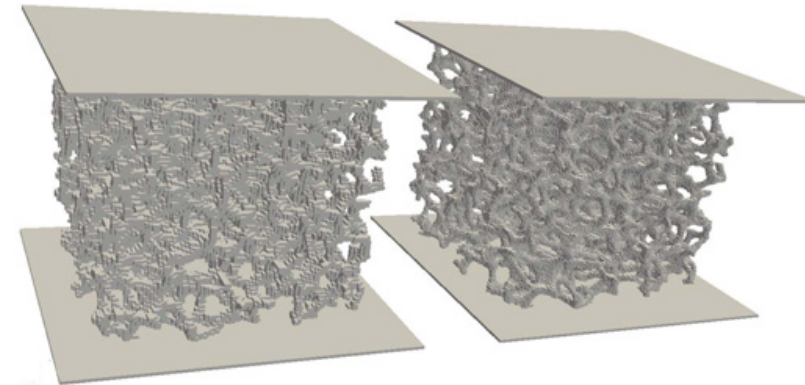

Figure 2. FEM meshes with element sizes of $170 \mu \mathrm{m}$ (left) and $85 \mu \mathrm{m}$ (right).

Table 1. Used material parameters for aluminium.

\begin{tabular}{lll}
\hline & Unit & Value \\
\hline Density & $\mathrm{kg} / \mathrm{m}^{3}$ & 2660 \\
Young's modulus & $\mathrm{N} / \mathrm{m}^{2}$ & $7 \mathrm{e} 10$ \\
Poisson ratio & - & 0.35 \\
Elastic limit & $\mathrm{N} / \mathrm{m}^{2}$ & $230 \mathrm{e} 6$ \\
Erosion (principal strain) & - & 0.1 \\
\hline
\end{tabular}

Similar investigations are done also for concrete [11], sand [12] and laminated glass [13].

The microstructure of the foam gained by micro-focus computer tomography $(\mu \mathrm{CT})$ is used in order to create a solid 3D mesh from the $\mu \mathrm{CT}$ hexahedron cell mesh. These meshes use quite a large number of elements. The meshes contain about 130,000 elements for a mesh size of $170 \mu \mathrm{m}$ and 5.4 million elements for a mesh size of $85 \mu \mathrm{m}$ (Fig. 2). While the coarser meshes are manageable, the calculation time for the finest mesh is very high. The coating thickness for the investigated foams is in the range between 25 and $75 \mu \mathrm{m}$, while the scanned one has a coating thickness of about $75 \mu \mathrm{m}$. Therefore, it is not possible to build up the coating in the raw resolution. Further approaches could use beam elements with much lower calculation times, for which the coating is defined via the cross section of the beam, similar to layered shell elements. Nevertheless, it will be quite complex to build beam element meshes basen on the scan results.

A quite generic elastic-plastic material law is used to represent the behaviour of the aluminium. No hardening is taken into account. In addition, an erosion criterion based on principal strain is introduced (Material parameters, see Table 1). The numerical virtual experiment is conducted displacement controlled. The displacements are introduced via rigid load plates similar to real experiments. Between all aluminium elements and these plates, a contact is defined by using a pinball method [14]. This very powerful method attaches spheres around each concerned element. The contact between these spheres is checked each step. In addition to the contact between the sample and the plates, a self contact is introduced in order to allow contact between the struts after larger displacements or failure of them.

The loading is applied in two different loading speeds: a moderate loading rate of $1 \mathrm{~m} / \mathrm{s}$ and a faster one of $10 \mathrm{~m} / \mathrm{s}$ are used. For a numerical specimen size of $14 \mathrm{~mm}$, these loading rates correspond to strain rates of 71 and 714 1/s resp. The objective of the virtual experiment is

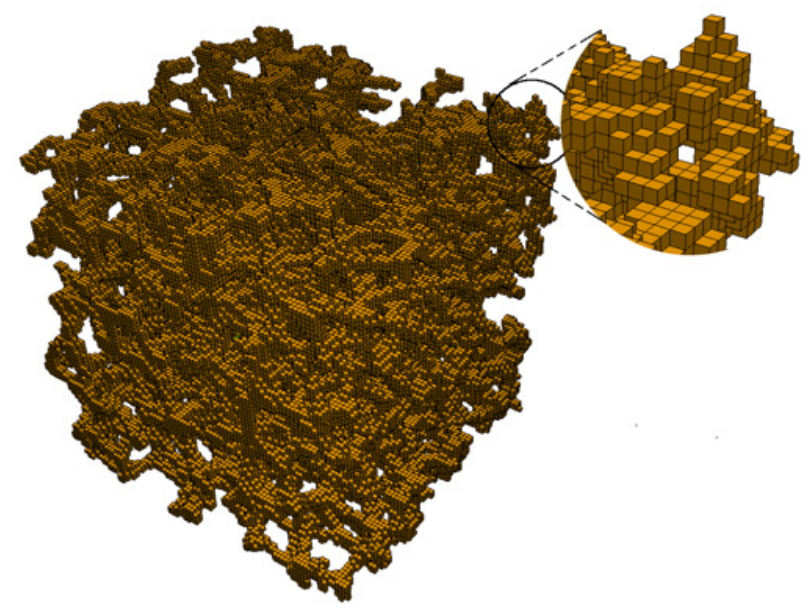

Figure 3. Voxel model of the foam. Each voxel was directly converted to a linear hexahedral element.

to obtain the macro stress-strain relation of a particular experiment. The strain can be calculated by dividing the actual displacement of the plates by the initial distance of the plates. The contact forces between the plate and the sample (aluminium hexahedrons) divided by the initial cross section of the sample provide the current stress.

\section{Results and discussions}

\subsection{Evaluation of the microstructure by means of micro-focus computer tomography}

For the development of the microstructural FE models, the radiograms were reconstructed with a cone-beam backprojection algorithm using Volex software (Fraunhofer-Allianz Vision, Germany). The resolution of the reconstructed three dimensional images was approximately $11.85 \mathrm{~mm}^{3}$. The reconstructed data were then used to develop the FE model for the simulation of the dynamic compression tests. The resolution of the original images $1445 \times 1459 \times 1330 \mathrm{px}$ was progressively reduced to $401 \times 401 \times 343 \mathrm{px}, 200 \times 200 \times 171 \mathrm{px}$ and $101 \times 101 \times 85 \mathrm{px}$ to lower the complexity of the FE model for the simulations. The resampled image data were used to develop microstructural voxel models using an in-house open source software tool [10] by directly converting every voxel (spatial pixel) into a linear hexahedral element (see Fig. 3).

\subsection{Experimental investigation of strain-rate dependency}

Figure 4 shows a comparison of the stress-strain diagrams obtained from the quasi-static and dynamic compression tests. For both strain-rates, there is a clearly visible increase in the plastic collapse stress (PCS), corresponding to the strength of the foams, and of the plateau stress with increasing coating thickness. Comparing one sample type/coating thickness at different strain-rates outlines that there is a pronounced strain-rate sensitivity for the coated foams, which becomes visible by increasing PCS and plateau stress and even by a change in the deformation mechanism. The change in the deformation mechanism 


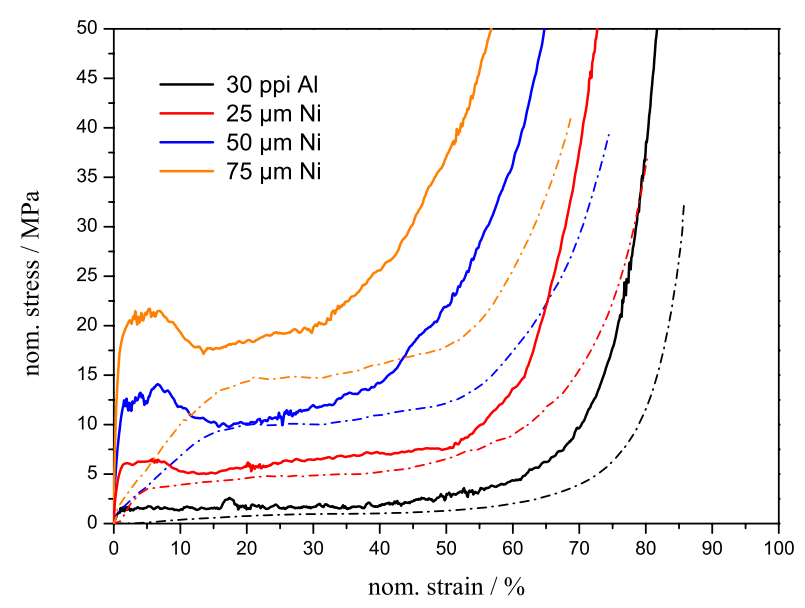

Figure 4. Comparison of the stress-strain diagrams for quasistatic and dynamic compression tests (dashed lines: quasi-static $5 \times 10^{-3} \mathrm{~s}^{-1}$, compact lines: dynamic $5000 \mathrm{~s}^{-1}$ ).

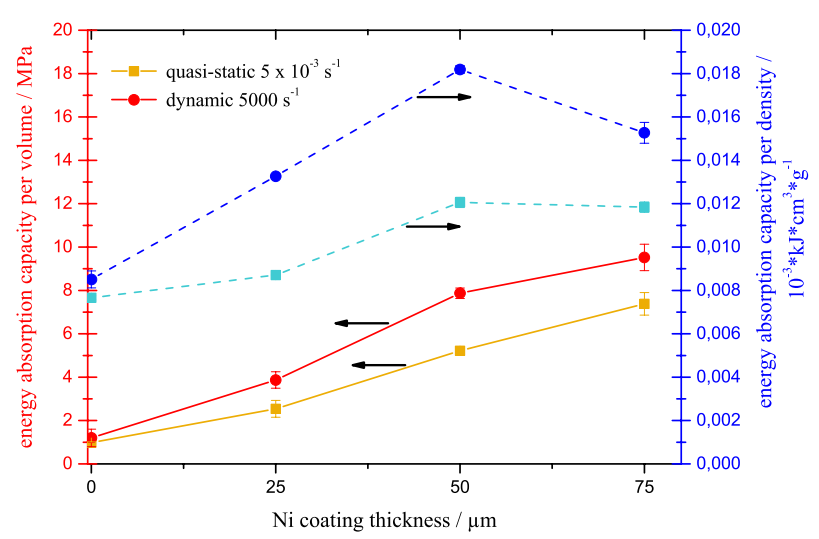

Figure 5. Specific energy absorption capacities up to the densification point as function of the $\mathrm{Ni}$ coating thickness, expressed per volume (lower curves) and per density (upper curves).

from quasi-static to dynamic mode is expressed by the pronounced plastic collapse peak in the dynamic curves. This comes from a time-delayed collapse of the pores, because, for high strain rates, the struts will first be stretched before they fail by buckling. The plateau stress is less strain-rate sensitive for small coating thicknesses. At higher coating thicknesses, rate effects occur. This rate dependency is also reflected in the energy absorption capacity, outlined in Fig. 5. For both strain rates, a linear increase of the energy absorption capacity per volume with increasing coating thickness can be observed. The rate dependency is pronounced by the higher energy absorption capacity under higher strain-rates. The pure aluminium foams show no strain-rate sensitivity, whereas the strain-rate sensitivity of the $\mathrm{Ni} / \mathrm{Al}$ hybrid foams increases with increasing coating thickness. For open-cell metal foams, strain rate dependency can only arise by micro-inertia effects and strain-rate sensitive strut material. In contrast to aluminium, nickel is strain-rate sensitive and hence, increasing the amount of nickel leads to a pronounced strain-rate dependency of the Ni/Al hybrid foams. The change in the deformation mechanism for the hybrid foams, expressed by the higher stiffness and the

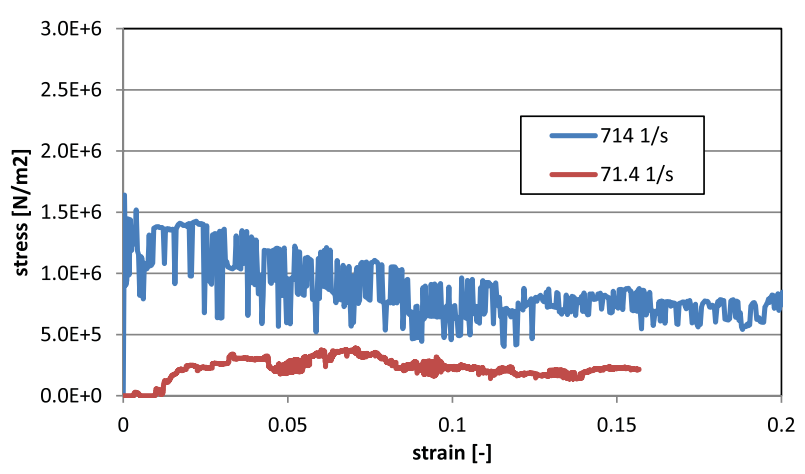

Figure 6. Influence of strain rate on stress-strain curve for coarse mesh $(170 \mu \mathrm{m})$ - detail for small strains.

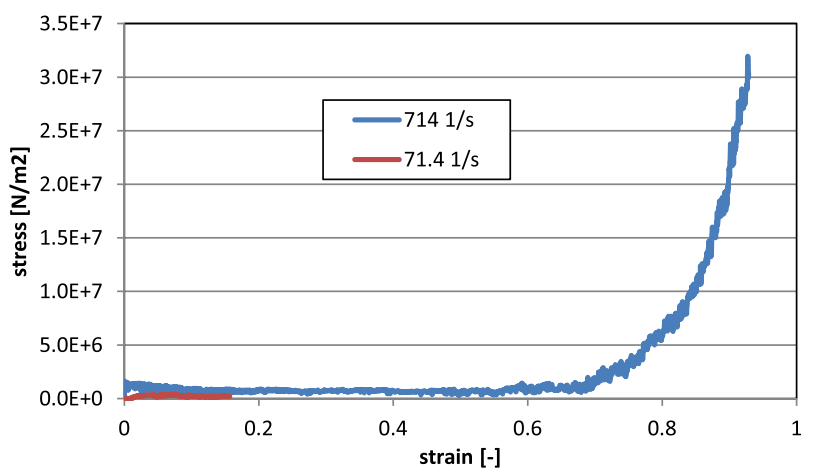

Figure 7. Influence of strain rate on stress-strain curve for coarse mesh $(170 \mu \mathrm{m})$.

appearance of a plastic collapse stress peak under dynamic load conditions, indicates that micro-inertia effects become important for the Ni/Al hybrid foams, but not for the pure aluminium foams.

\subsection{Numerical investigation of strain-rate dependency}

The first calculation with the smaller strain rate is run up to a displacement of about $2 \mathrm{~mm}$ corresponding to a strain of about 0.15 is reached. The classical foam behaviour can be observed (Fig. 6). After a linear increase of the stress, a plateau in the stress-strain curve can be observed. The calculation is not done long enough to also see the steepening up of the curve. The calculation was performed on 6 processors of a very powerful desktop PC and took about 12 days.

The calculation with a strain rate of about 714 1/s was done until a much higher strain was reached. It can be observed (see Figs. 6 and 7) that the stress increases much faster at the beginning of the stress-strain relation. In addition, the curve is much more scattered due to the oscillations of the loading wave. Figure 7 shows also that the model can represent quite well the steepening up of the stress-strain curve after a certain point.

Figure 8 shows the displacement history of the virtual experiment. It can be observed that, after the first $0.25 \mathrm{~ms}$, some of the struts have failed and the first strut layers are compacted. This mechanism proceeds unit about $1 \mathrm{~ms}$. At that time, the sample has somehow reached the point at which the stiffness is increasing again. At $1.25 \mathrm{~ms}$, the 


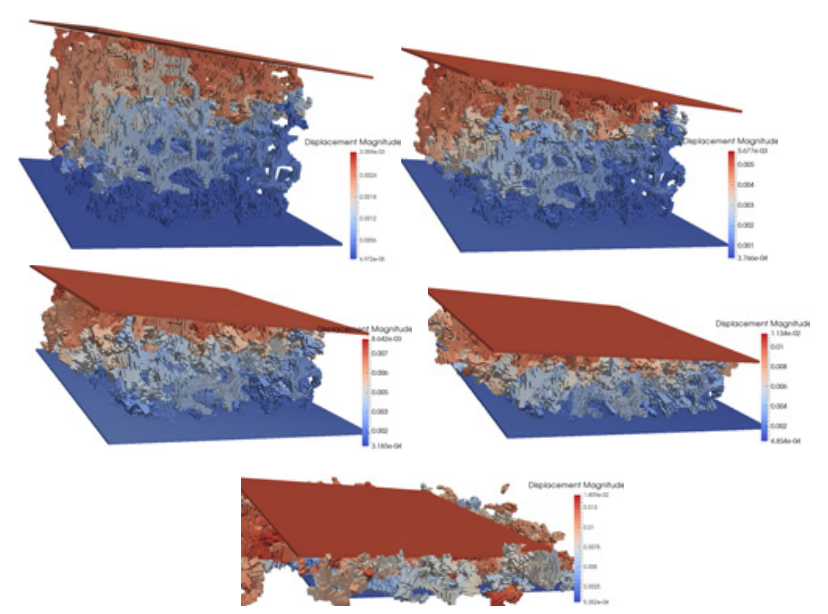

Figure 8. Displacement history for strain-rate of about 714 1/s: $0.25 \mathrm{~ms}, 0.5 \mathrm{~ms}, 0.75 \mathrm{~ms}, 1.0 \mathrm{~ms}, 1.25 \mathrm{~ms}$.

sample is strongly compacted. The stiffness of the material should not be higher than the one from pure aluminium, which is still the case. Further calculation is very time consuming due to the large number of contacts between all elements.

\section{Conclusion}

In this contribution, the strain-rate dependence of pure aluminium and $\mathrm{Ni} / \mathrm{Al}$ hybrid metal foams was studied by experimental und numerical dynamic compression tests. The SHPB tests outline a significant strain-rate sensitivity for the hybrid foams, which increases with the coating thickness, but not for the aluminium foams. For the evaluation of a micro model as virtual testing laboratory, $\mu \mathrm{CT}$ measurements were performed to determine the microstructure of the foams, since micro-inertia effects comming from the microstructure are crucial for strainrate dependence. The numerical simulations show a strainrate sensitivity for the alumminium foams, too. Hence, the strain-rate dependence of the $\mathrm{Ni} / \mathrm{Al}$ hybrid foams results on the one hand from micro-inertia effects and mainly on the other hand from the strain-rate sensitivity of the coating metal nickel.

\section{References}

[1] M.F. Ashby, Metall. Trans. A 14, 1755-1769 (1983)

[2] M.F. Ashby, A. Evans, N.A. Fleck, L.J. Gibson, J.W. Hutchinson, H.N.G. Wadley, Metal foams: design guide (Butterwoth-Heinemann, Oxford, 2000) 1-40

[3] Y. Boonyongmaneerat, C.A. Schuh, D.C. Dunand, Scripta Mater. 59, 336-339 (2008)

[4] B.A. Bouwhuis, J.L. McCrea, G. Palumbo, G.D. Hibbard, Acta Mater. 57, 4046-4053 (2009)

[5] A. Jung and M.R. Koblischka and E. Lach and S. Diebels and H. Natter, Int. J. Mater. Sci. 2, 97-107 (2012)

[6] A. Jung, H. Natter, R. Hempelmann, S. Diebels, M. R. Koblischka, U. Hartmann E. Lach, ECS Transactions 25, 165-172 (2010)

[7] A. Jung, H. Natter, S. Diebels, E. Lach R. Hempelmann, Adv. Eng. Mater. 13, 23-28 (2011)

[8] A. Jung and H. Natter and R. Hempelmann and E. Lach, Metal foams, EP 2261398 A1

[9] D. Vavrik and J. Jakubek, Nucl. Instrum. Meth. A. 607, 212-214 (2009)

[10] O. Jirousek, T. Doktor, D. Kytyr, P. Zlamal, T. Fila, P. Koudelka, I. Jandejsek and D. Vavrik, J. Instrum. 8(2), C02012 (2013)

[11] W. Riedel, M. Wicklein, K. Thoma, Int. J. Impact Eng. 35(3), 155-171 (2008)

[12] M. Larcher, N. Gebbeken, EPJ Web of Conferences 26 (2012)

[13] Martin Larcher, George Solomos, Folco Casadei, Norbert Gebbeken, Int. J. Impact Eng. 39, 42-50 (2012)

[14] F. Casadei. A Hierarchic Pinball Method for Contact-Impact in Fast Transient Dynamics. VI Congresso Nazionale della Societ'a Italiana di Matematica Applicata e Industriale (SIMAI 2002), Chia (Cagliari), Italy, 27-31 May 2002 